\title{
VAC_19 - System biology analysis of THP1 cell line as in vitro model to evaluate yellow fever vaccine
}

Andréa Marques Vieira da Silva ${ }^{*}$; Tamiris Azamor da Costa Barros²; Thyago Leal Calvo ${ }^{3}$; Juliana Gil

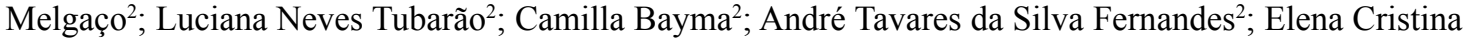
Caride $^{2}$; Milton Ozório Moraes ${ }^{3}$; Ana Paula Dinis Ano Bom² .

${ }^{1}$ Fiocruz/COC;

${ }^{2}$ Fiocruz/Bio-Manguinhos;

${ }^{3}$ Fiocruz/IOC.

Introduction: Bio-Manguinhos is the world's biggest producer of the attenuated yellow fever vaccine (YFV17DD), a centennial product recognized as one of the most successful vaccines ever developed in terms of efficacy and safety. Besides, it is considered the standard vaccine to investigate the dynamics and quality of an ideal vaccine. Although, YFV17DD present rare cases of adverse events post-vaccination, Bio-Manguinhos has been seeking new vaccine design strategies that achieve the necessary safety in AEPV risk groups. The production of new immunobiologicals is no longer empirical and has been improved by technical-scientific approaches, demanding deeper interdisciplinary studies in different fields such as genetics and immunology. In this context, systems biology has been increasingly relevant and could help to design new vaccines.

Objective: To establish an analytical platform for gene expression in systems biology for in vitro evaluation of Yellow Fever candidate vaccines developed in Bio-Manguinhos using the YFV17DD vaccine as standard.

Methodology: Plates with 24 wells contained $1 \times 10^{6}$ cells of the monocytic human THP1 cell line maintained in RPMI medium containing 10\% fetal bovine serum. Cells were infected with YFV attenuated (AYFV) MOI 0.002, stimulated with $1 \mu \mathrm{g} / \mathrm{mL}$ of envelope protein E of YFV (YFE), or maintained with RPMI medium (mock) for 48 hours. Then, the culture total RNA was extracted, viral RNA detected by $\mathrm{qPCR}$, and the cellular RNA were prepared for RNAseq using a Truseq RNA sample preparation kit (Illumina ${ }^{\circledR}$ ) according to manufacturer's recommendations. Next, monocyte activation was evaluated by immunophenotyping using the CD11a, CD14, CD16 and HLA-DR markers. From the supernatant chemokines CCL3, CCL5, and CXCL8, were quantified using liquid microarray assay.

Results: Functional analysis demonstrated that THP1 model was susceptible to viral replication, and presented a differential activation profile when comparing to mock condition: YFA lead to CD14+CD11a+ profile (monocytic dendritic cells), while those stimulated with YFE showed the activated non-classical profile CD14+CD16+ (pro-inflammatory monocytes). Both yellow fever vaccine approaches was able to induce production of cytokines such as CCL3 and CXCL8 in THP1 model. According RNAseq analysis the YFA infected cells presented significant high expression of genes related with the processes: virus and inflammatory response, regulation of immune effector process, response to external stimulus, leukocyte activation, and innate immune response. Otherwise, YFE stimulated cells response was characterized by leukocyte migration, lymphocyte activation, monocyte chemotaxis, and induction of adaptive immune response.

Conclusion: Taking together functional and transcriptomics analysis, here we demonstrated that YFA lead to a strong innate and inflammatory response, whether YFE stimulated a mild innate response along with a faster induction of genes related to induction of adaptative response in the THP1 model. The molecular and functional parameter stipulated here could contribute to screening vaccines under development, contributing to minimize animal model use.

Keywords: In vitro model; Systems biology; Yellow fever vaccine 